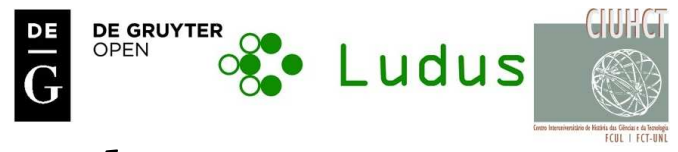

\title{
Games and Puzzles
}

\section{INDEPENDENCE AND DOMINATION ON SHOGIBOARD GRAPHS}

\author{
Doug Chatham \\ Morehead State University, Department of Mathematics and Physics \\ d. chatham@moreheadstate.edu
}

\begin{abstract}
Given a (symmetrically-moving) piece from a chesslike game, such as shogi, and an $n \times n$ board, we can form a graph with a vertex for each square and an edge between two vertices if the piece can move from one vertex to the other. We consider two pieces from shogi: the dragon king, which moves like a rook and king from chess, and the dragon horse, which moves like a bishop and rook from chess. We show that the independence number for the dragon kings graph equals the independence number for the queens graph. We show that the (independent) domination number of the dragon kings graph is $n-2$ for $4 \leqslant n \leqslant 6$ and $n-3$ for $n \geqslant 7$. For the dragon horses graph, we show that the independence number is $2 n-3$ for $n \geqslant 5$, the domination number is at most $n-1$ for $n \geqslant 4$, and the independent domination number is at most $n$ for $n \geqslant 5$.
\end{abstract}

Keywords: shogi, $n$-queens problem, combinatorics.

\section{Introduction}

Hundreds of papers have been written on problems involving the placement of chess pieces on a board so that the placement satisfies given constraints 11, 6, 10, One famous example is the $n$-queens problem of placing the maximum number of queens on an $n \times n$ chessboard so that no queen "attacks" any other queen (i.e., no queen can reach the position of another queen in one move). Another problem, the queens domination problem, calls for placing the minimum number of queens on an $n \times n$ board so that each empty square is attacked by at least one queen. A third example, the queens independent domination problem, calls for the placement of the minimum number of queens necessary on an $n \times n$ board so that no two queens attack each other and every empty square is attacked by at least one queen.

Among other approaches, these placement problems have been framed and studied as problems in graph theory. Suppose $M$ is a piece in a chesslike game played on an $n \times n$ board and suppose its set of legal moves form a 
symmetric relation on the set of positions; i.e., if $M$ can move from position $a$ to position $b$ it can also move from $b$ to $a$ (we exclude the case where $M$ is a chess pawn, which only moves in forward directions). Then we define the pieces graph $M_{n}$ to have vertex set $V\left(M_{n}\right)=\{(i, j) \mid i, j \in\{0, \ldots, n-1\}\}$, with $(i, j)$ representing the square in column $i$ and row $j$, and edge set $E\left(M_{n}\right)=\{\{(a, b),(i, j)\} \mid M$ can move from $(a, b)$ to $(i, j)\}$. For example, since the rook attacks all squares in its row and column, the rooks graph $R_{n}$ has edge set $E\left(R_{n}\right)=\{\{(a, b),(i, j)\} \mid a=i$ or $b=j\}$. Also, since the bishop attacks all squares in its "falling diagonal" (i.e., squares for which the sum of row and column coordinates is a constant) and its "rising diagonal" (i.e., squares for which the row coordinate minus the column coordinate is a constant), the bishops graph $B_{n}$ has edge set $E\left(B_{n}\right)=\{\{(a, b),(i, j)\} \mid a+b=i+j$ or $a-b=i-j\}$. Finally, since the queen combines the powers of rook and bishop, the edge set of the queens graph is $E\left(Q_{n}\right)=E\left(R_{n}\right) \cup E\left(B_{n}\right)$.

Recall from [4] that in any graph $G$, two vertices are adjacent if they share an edge, a set of vertices of $G$ is independent if no two of the vertices in that set are adjacent, and a set of vertices is a dominating set if each vertex of $G$ is either in the set or adjacent to an element of the set. For any graph $G$ we define the following domination parameters:

- the independence number $\beta(G)$ is the maximum cardinality of an independent set of vertices of $G$

- the domination number $\gamma(G)$ is the minimum cardinality of a dominating set of vertices of $G$

- the independent domination number $i(G)$ is the minimum cardinality of an independent dominating set of vertices of $G$.

These parameters are related by an inequality chain: for any graph $G$, we have $\gamma(G) \leqslant i(G) \leqslant \beta(G)$ [4, Corollary 3.7].

The values of these parameters for chessboard graphs are known for, among other pieces, the rook and bishop: $\beta\left(R_{n}\right)=i\left(R_{n}\right)=\gamma\left(R_{n}\right)=n, \beta\left(B_{n}\right)=2 n-2$ for $n \geqslant 2$, and $i\left(B_{n}\right)=\gamma\left(B_{n}\right)=n$. Also, $\beta\left(Q_{n}\right)=n$ for $n \geqslant 4\left(\beta\left(Q_{n}\right)=1\right.$ for $n=1$ or 2 , and 2 for $n=3)$. However, the values of $i\left(Q_{n}\right)$ and $\gamma\left(Q_{n}\right)$ are known for only finitely many $n$ [5. Chapter 6].

In this paper we consider two pieces from shogi, a Japanese relative of chess: the dragon king, which can move one square diagonally or any number of squares vertically or horizontally, and the dragon horse, which can move any number of squares diagonally or one square vertically or horizontally. (For more information on shogi, we refer the interested reader to [2].) Let $D_{n}$ be the dragon kings graph on the $n \times n$ board - so $E\left(D_{n}\right)=\{\{(a, b),(c, d)\} \mid a=c$ or $b=d$ or $\max (|a-c|,|b-d|)=1\}$. Also, let $H_{n}$ be the dragon horses graph on the $n \times n$ board - so $E\left(H_{n}\right)=\{\{(a, b),(c, d)\} \mid a+c=b+d$ or $a-c=b-d$ or $\max (|a-c|,|b-d|)=1\}$. We note that $E\left(D_{n}\right) \cup E\left(H_{n}\right)=E\left(Q_{n}\right)$, so exploring domination parameters for the dragon kings graph and the dragons horses graph may provide insight into the problems of queens domination and queens independent domination. 
In Section \we determine $\beta\left(D_{n}\right), \gamma\left(D_{n}\right)$, and $i\left(D_{n}\right)$. In Section 2 we determine $\beta\left(H_{n}\right)$ and find upper bounds for $\gamma\left(H_{n}\right)$ and $i\left(H_{n}\right)$. We also discuss computer calculations for $\gamma\left(H_{n}\right)$ and $i\left(H_{n}\right)$. In Section [3] we discuss open problems and avenues for further study.

\section{Dragon kings}

First we show that the dragon kings independence number equals the queens independence number.

Proposition 1. For $2 \leqslant n \leqslant 3, \beta\left(D_{n}\right)=\beta\left(Q_{n}\right)=n-1$. For all other values of $n, \beta\left(D_{n}\right)=\beta\left(Q_{n}\right)=n$.

Proof: It is easy to check that $\beta\left(D_{2}\right)=1=\beta\left(Q_{2}\right)$ and $\beta\left(D_{3}\right)=2=\beta\left(Q_{3}\right)$, so suppose $n \neq 2$ and $n \neq 3$. Since $E\left(R_{n}\right) \subseteq E\left(D_{n}\right) \subseteq E\left(Q_{n}\right)$, we have $\beta\left(R_{n}\right) \geqslant \beta\left(D_{n}\right) \geqslant \beta\left(Q_{n}\right)$. So $n=\beta\left(R_{n}\right) \geqslant \beta\left(D_{n}\right) \geqslant \beta\left(Q_{n}\right)=n$, and therefore $\beta\left(D_{n}\right)=\beta\left(Q_{n}\right)=n$ for $n=1$ and $n \geqslant 4$.

The reader might wonder what the maximum independent sets of $D_{n}$ are. For $n \geqslant 4$, we obtain a maximum independent set of dragon kings on the squares $(i, \sigma(i)), i=0, \ldots n-1$, where $\sigma$ is any permutation of $\{0, \ldots, n-1\}$ such that $\sigma(i+1) \neq \sigma(i) \pm 1$ for $i=0, \ldots, n-2$. Such permutations exist; for example, consider $\{(i,(2 i+1) \bmod n) \mid i=0, \ldots, n-1\}$ for $n \geqslant 4$ (as pictured in Figure 11). These permutations have been studied in many settings, and the sequence $\{a(n)\}_{n=1}^{\infty}$, where $a(n)$ is the number of such permutations of $\{0, \ldots, n-1\}$, is the sequence A002464 of the OEIS [8].

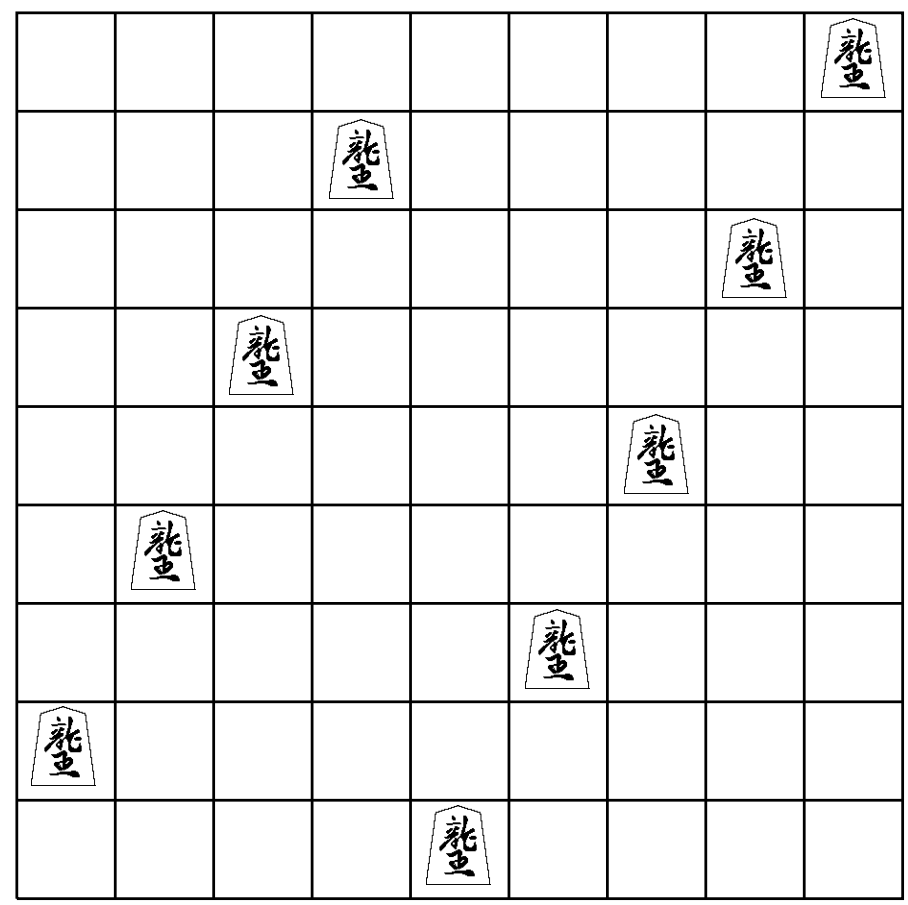

Figure 1: A $9 \times 9$ board with 9 independent dragon kings. 
Next we consider the domination and independent domination numbers. For $1 \leqslant n \leqslant 3, i\left(D_{n}\right)=\gamma\left(D_{n}\right)=1$. In the next few theorems we show that the domination number and the independent domination number of the dragon kings graph are equal for all $n$.

Lemma 2. Suppose we have an $n \times n$ board dominated by $k$ independent dragon kings. Then we can dominate an $(n+1) \times(n+1)$ board by $k+1$ independent dragon kings.

Proof: We note that at least one of the four corner squares is empty, since otherwise the dragon kings are not all independent. Without loss of generality, say that square is $(n-1, n-1)$. (If not, we can rotate until it is.) Add row $n$ and column $n$ and place a dragon king on square $(n, n)$. The new dragon king dominates the newly added squares and attacks no other dragon king.

Proposition 3. For $3 \leqslant n \leqslant 6, \gamma\left(D_{n}\right)=i\left(D_{n}\right)=n-2$.

Proof: First we show $\gamma\left(D_{n}\right) \leqslant i\left(D_{n}\right) \leqslant n-2$. For a $3 \times 3$ board, place a dragon king in the center square and note that all other squares are attacked. We can now prove our claim by induction on $n$ using Lemma 2 .

To complete the proof it remains to show $n-2 \leqslant \gamma\left(D_{n}\right)$ for $3 \leqslant n \leqslant 6$. This is obviously true for $n=3$, so let $n \geqslant 4$ and suppose we have a dominating set $A$ for $D_{n}$ of size $n-3$. We have at least three empty columns (that is, columns containing no elements of $A$ ) and at least three empty rows.

Let $c_{1}<c_{2}<c_{3}$ indicate the numbers of three empty columns and $r_{1}<r_{2}<r_{3}$ indicate the numbers of three empty rows. Consider squares $\left(c_{1}, r_{1}\right),\left(c_{1}, r_{3}\right)$, $\left(c_{3}, r_{1}\right)$, and $\left(c_{3}, r_{3}\right)$. Each of those squares must be attacked diagonally by members of $A$. For any $i$, the squares in column $c_{i}$ (respectively, row $r_{i}$ ) can only be diagonally attacked by pieces in column $c_{i}-1$ or $c_{i}+1$. (respectively, row $r_{i}-1$ or $r_{i}+1$.) We show that no single piece covers any pair of the squares under consideration. If a dragon king attacked both $\left(c_{1}, r_{1}\right)$ and $\left(c_{1}, r_{3}\right)$ diagonally, that piece would be in row $r_{1}+1=r_{3}-1$. But that row would then also be row $r_{2}$, which is empty. So the dragon king attacking $\left(c_{1}, r_{1}\right)$ must be distinct from the dragon king attacking $\left(c_{1}, r_{3}\right)$. Similar arguments work for any other pair of the considered squares. Hence our dominating set must have at least 4 elements. But for $4 \leqslant n \leqslant 6, n-3<4$, a contradiction. We must have $n-2 \leqslant \gamma\left(D_{n}\right)$ for $n=4,5,6$.

An anonymous reviewer of a previous draft of this paper kindly provided the following proof:

Proposition 4. (Anonymous) For $n \geqslant 7, n-3 \leqslant \gamma\left(D_{n}\right) \leqslant i\left(D_{n}\right)$. 


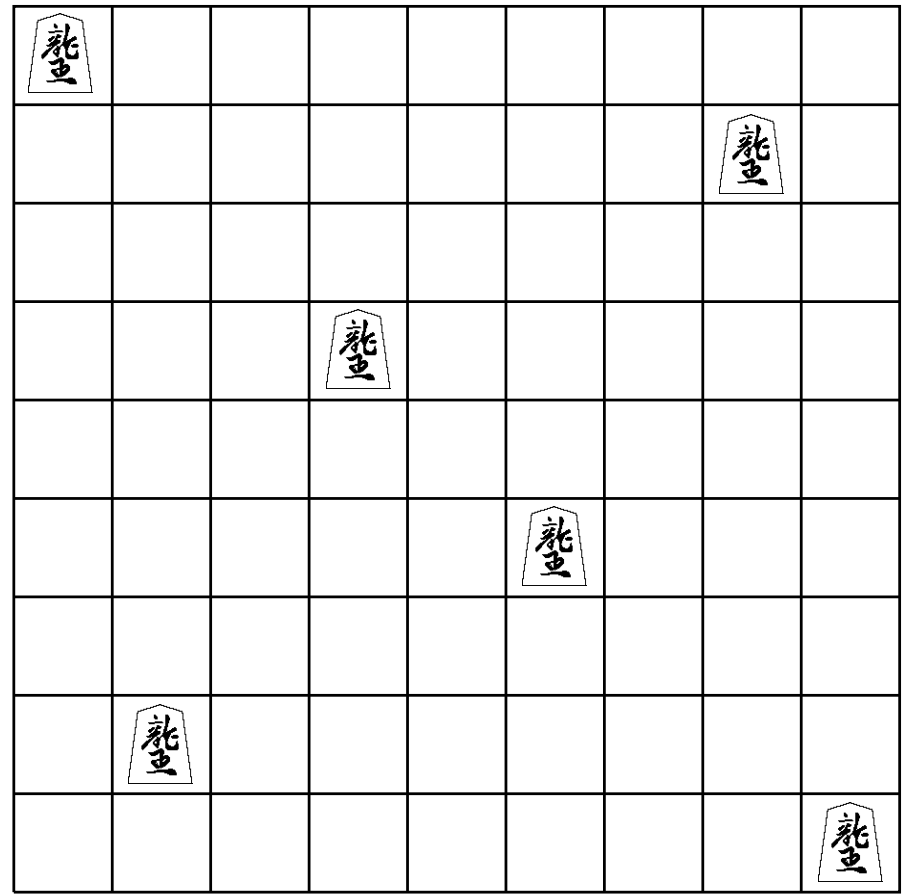

Figure 2: A $9 \times 9$ board dominated by 6 independent dragon kings.

Proof: Suppose that for some integer $n \geqslant 7$ there exists a dominating set $A$ of size $n-4$ for $D_{n}$. Let $e$ denote the number of empty rows and let $f$ denote the number of empty columns. By rotating the board if necessary, we may assume that $e \leqslant f$.

For $i=0,1,2$ let $A_{i}=\{s \in A$ : square $s$ is in a row adjacent to exactly $i$ empty rows $\}$ and let $a_{i}=\left|A_{i}\right|$. Then

$$
a_{0}+a_{1}+a_{2}=n-4 .
$$

For $i=0,1,2$, let $d_{i}$ be the number of rows having a square in $A_{i}$. Then

$$
d_{0}+d_{1}+d_{2}=n-e .
$$

The definitions imply $d_{i} \leqslant a_{i}$ for each $i$, so (II) and (2) give $e-4$ as a sum of nonnegative integers:

$$
\left(a_{0}-d_{0}\right)+\left(a_{1}-d_{1}\right)+\left(a_{2}-d_{2}\right)=e-4 .
$$

Since at most $2 e$ rows are adjacent to empty rows,

$$
d_{1}+2 d_{2} \leqslant 2 e .
$$

Let $U$ be the set of board squares not in the same row or column as any square of $A$; these squares must be covered diagonally. There are ef squares in $U$. 
For $i=1,2$, a square of $A_{i}$ covers at most $2 i$ squares of $U$, and squares of $A_{0}$ cover no squares of $U$. Thus $2 a_{1}+4 a_{2} \geqslant e f$. We rewrite this as

$$
2\left[d_{1}+\left(a_{1}-d_{1}\right)\right]+4\left[d_{2}+\left(a_{2}-d_{2}\right)\right] \geqslant \text { ef } .
$$

By (44), $4 e \geqslant 2 d_{1}+4 d_{2}$, so (5) implies

$$
4 e+2\left(a_{1}-d_{1}\right)+4\left(a_{2}-d_{2}\right) \geqslant e f .
$$

From (B3), $4(e-4) \geqslant 4\left(a_{1}-d_{1}\right)+4\left(a_{2}-d_{2}\right) \geqslant 2\left(a_{1}-d_{1}\right)+4\left(a_{2}-d_{2}\right)$, so $4 e+4(e-4) \geqslant e f$. This gives

$$
0 \geqslant e(f-e)+(e-4)^{2} .
$$

Since each of $e, f-e$, and $(e-4)^{2}$ is nonnegative, (6i) implies $e=f=4$. Then (3) implies $a_{i}=d_{i}$ for $i=0,1,2$ : every nonempty row contains just one square of $A$. Since $e=f$, we may similarly conclude that every nonempty column contains just one square of $A$.

Let $c_{1}<c_{2}<c_{3}<c_{4}$ be the numbers of the empty columns, and $r_{1}<r_{2}<r_{3}<r_{4}$ be the numbers of the empty rows. The four squares of $U$ in row $r_{1}$ must be diagonally covered by squares of $A$ in rows $r_{1}+1$ and $r_{1}-1$. Then these rows must contain exactly one square each of $A$, and these must be in columns $c_{1}+1$ and $c_{3}+1$, with $c_{2}=c_{1}+2$ and $c_{4}=c_{3}+2$. However, the four squares of $U$ in row $r_{4}$ will similarly need to be covered by squares of $A$ in columns $c_{1}+1$ and $c_{3}+1$, and these squares cannot be the same as those covering the squares of $U$ in row $r_{1}$. This means $A$ contains two squares in some columns, a contradiction. Thus no dominating set of size $n-4$ exists.

Corollary 5. For $n \geqslant 7, \gamma\left(D_{n}\right)=i\left(D_{n}\right)=n-3$.

Proof: By Proposition \&, it suffices to show an independent dominating set of $D_{n}$ for each $n \geqslant 7$. For $n=7$, take an $7 \times 7$ board and place dragon kings on squares $(0,0),(2,4),(4,2)$ and $(6,6)$. We can check that this set is an independent dominating set. Then we can prove the statement by induction on $n$ using Lemma 2] (an example of the construction is shown in Figure 21).

\section{Dragon horses}

We start the section by determining the independence number of the dragon horses graph.

Proposition 6. The independence number of the dragon horses graph, $\beta\left(H_{n}\right)$, is 1 for $n=1$ and $n=2, n$ for $n=3$ and $n=4$, and $2 n-3$ for $n \geqslant 5$.

Proof: On a $1 \times 1$ or a $2 \times 2$ board, a single dragon horse placed on any square leaves all other empty squares attacked, so $\beta\left(H_{1}\right)=\beta\left(H_{2}\right)=1$.

On a $3 \times 3$ board, we can place dragon horses on $(0,1),(2,0)$ and $(2,2)$ to show $\beta\left(H_{3}\right) \geqslant 3$. To see $\beta\left(H_{3}\right) \leqslant 3$, suppose 4 pieces are on the board. If they are not on the four corners, then at least two are on physically touching squares, and if the pieces are on the four corners, then we have pieces on the same diagonal.

Recreational Mathematics Magazine, Number 8, pp. 25-37 DOI: https://doi.org/10.1515/rmm-2017-0018 
To see that $\beta\left(H_{4}\right) \geqslant 4$, place dragon horses on squares $(0,0),(0,2),(2,1)$, and $(2,3)$. To see that $\beta\left(H_{4}\right) \leqslant 4$, partition the board into a $2 \times 2$ array of $2 \times 2$ blocks and note that each block can only hold one dragon horse of an independent set.

For $n \geqslant 5$, we show $\beta\left(H_{n}\right) \geqslant 2 n-3$. If $n$ is odd, place dragon horses on $\left(\frac{n-1}{2}, \frac{n-1}{2}\right), \quad(1,0), \quad(1, n-1)$, and, for each $i=1, \ldots, \frac{n-3}{2}, \quad(0,2 i)$, $(n-1,2 i),(2 i+1,0)$, and $(2 i+1, n-1)$. If $n$ is even, place dragon horses on $(0,0),(n-2,0),(1, n-1),(n-1, n-2),\left(\frac{n}{2}-1, \frac{n}{2}\right)$, and, for each $i=1, \ldots, \frac{n}{2}-2$, $(2 i, 0),(2 i+1, n-1),(0,2 i+1)$, and $(n-1,2 i)$. Examples of these constructions are pictured in Figures [3 and 4. We can check that none of these positions are on the same diagonal and that for any pair of positions, either the row coordinates or the column coordinates differ by at least 2 .

To conclude the proof, we show $\beta\left(H_{n}\right) \leqslant 2 n-3$ for $n \geqslant 5$. Partition the board into $2 n-3$ "slices", where the first slice consists of squares $(0,0),(0,1)$, and $(1,0)$, the $(2 n-3)^{r d}$ slice consists of squares $(n-1, n-2),(n-2, n-1)$, and $(n-1, n-1)$, and the $i^{t h}$ slice for $i=2, \ldots 2 n-2$ consists of the squares in the $i^{\text {th }}$ falling diagonal. Each slice can have at most one independent dragon horse, so the board can have at most $2 n-3$ independent dragon horses.

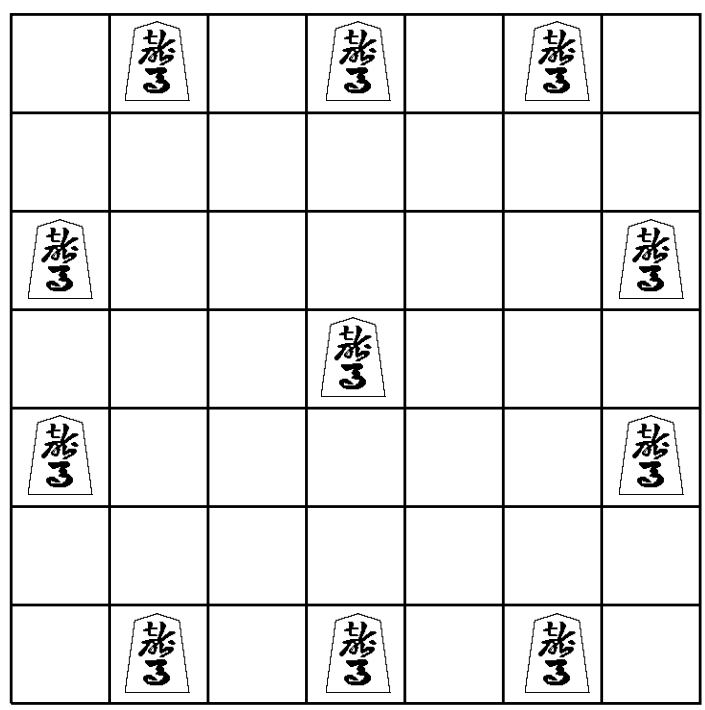

Figure 3: A $7 \times 7$ board with 11 independent dragon horses.

Next we consider the domination and independent domination numbers. We note that for $1 \leqslant n \leqslant 3, i\left(H_{n}\right)=\gamma\left(H_{n}\right)=1$. We obtain an upper bound for the domination number of the dragon horses graph. 


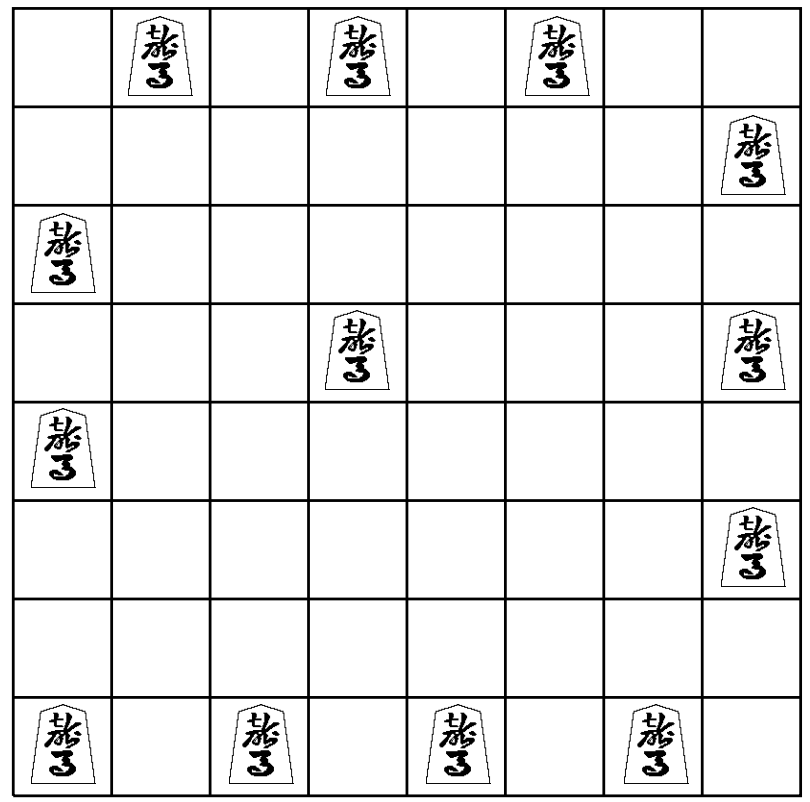

Figure 4: An $8 \times 8$ board with 13 independent dragon horses.

Proposition 7. For $n \geqslant 4, \gamma\left(H_{n}\right) \leqslant n-1$.

Proof: If $n$ is odd, we place dragon horses on all but the bottom row of the central column, as shown in Figure [5, and check that the empty squares are dominated. If $n=2 k$ is even, we place dragon horses on all but the first and last rows of column $k$ and square $(n-1, k)$, as shown in Figure 6, and check that the empty squares are dominated.

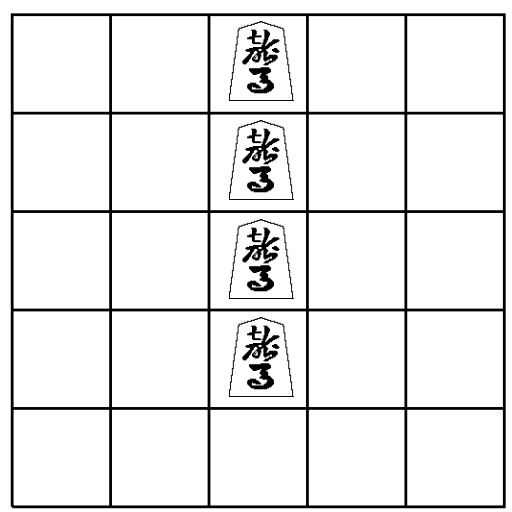

Figure 5: A $5 \times 5$ board dominated by 4 dragon horses.

We next obtain an upper bound for the independent domination number of the dragon horses graph. 


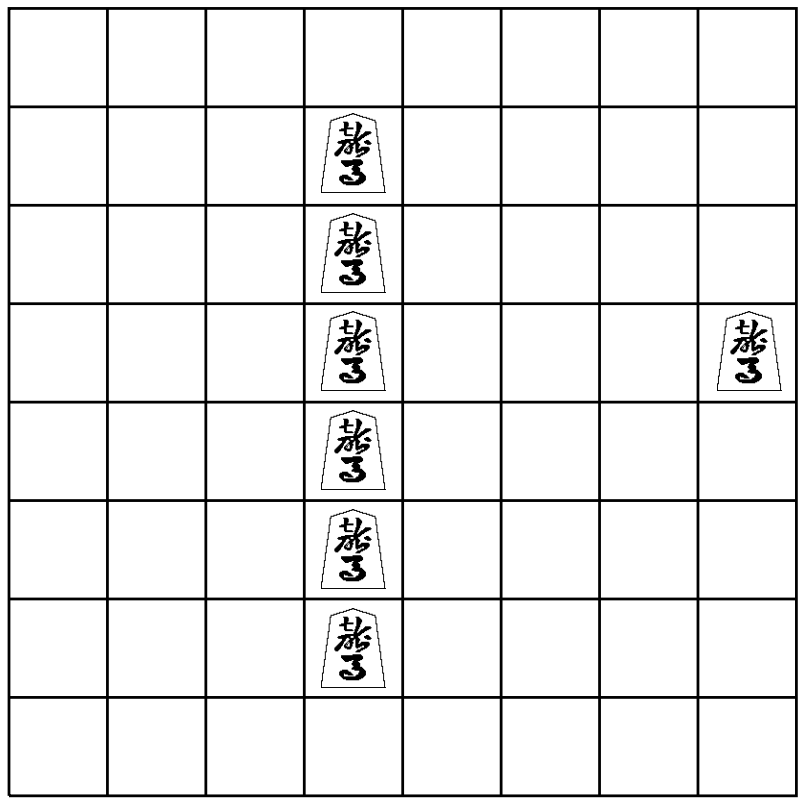

Figure 6: A $8 \times 8$ board dominated by 7 dragon horses.

Proposition 8. For $n \geqslant 5, i\left(H_{n}\right) \leqslant n$.

Proof: If $n$ is odd, place dragon horses on squares $(1,2 i+1)$ and $(n-2,2 i)$ for each $i$ such that $0 \leqslant i \leqslant \frac{n-1}{2}-1$ and square $(n-2, n-1)$, as shown in Figure 7]. If $n$ is even, place dragon horses on squares $(1,2 i)$ and $(n-2,2 i)$ for each $i$ such that $0 \leqslant i \leqslant \frac{n}{2}-1$, as shown in Figure [ In each case, we can check that each empty square is dominated and that none of the dragon horses attack each other.

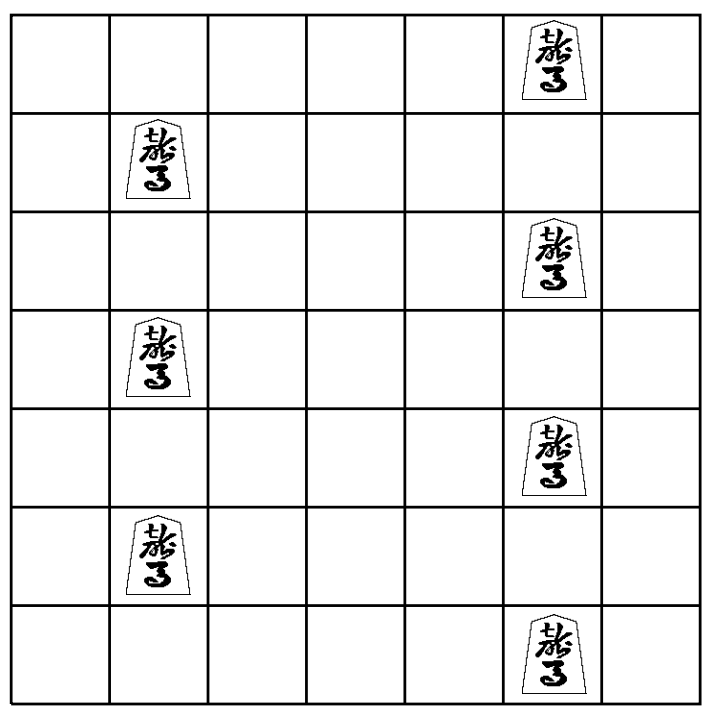

Figure 7: A $7 \times 7$ board dominated by 7 independent dragon horses. 


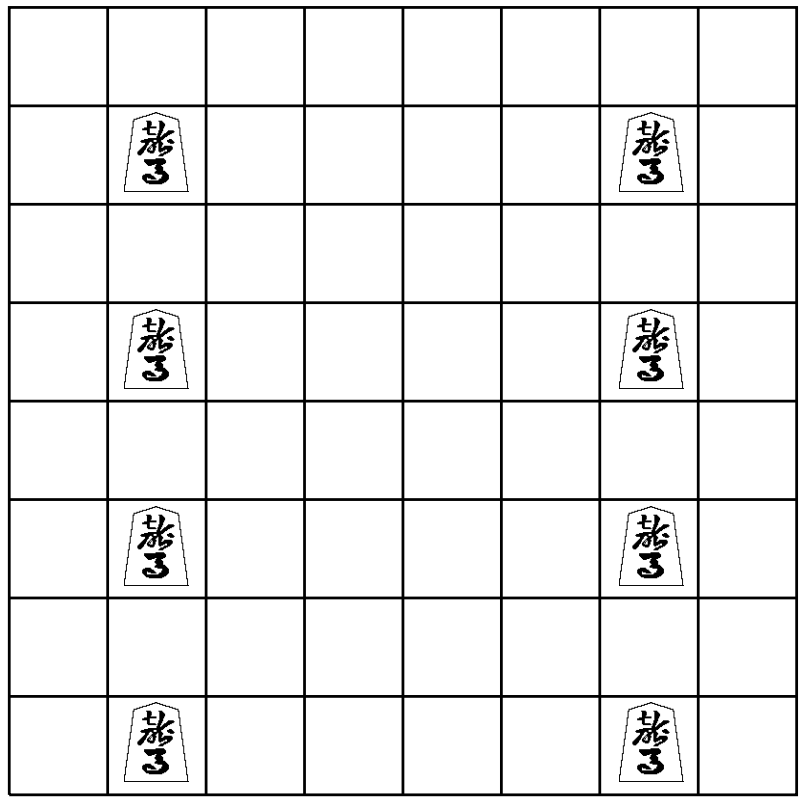

Figure 8: A $8 \times 8$ board dominated by 8 independent dragon horses.

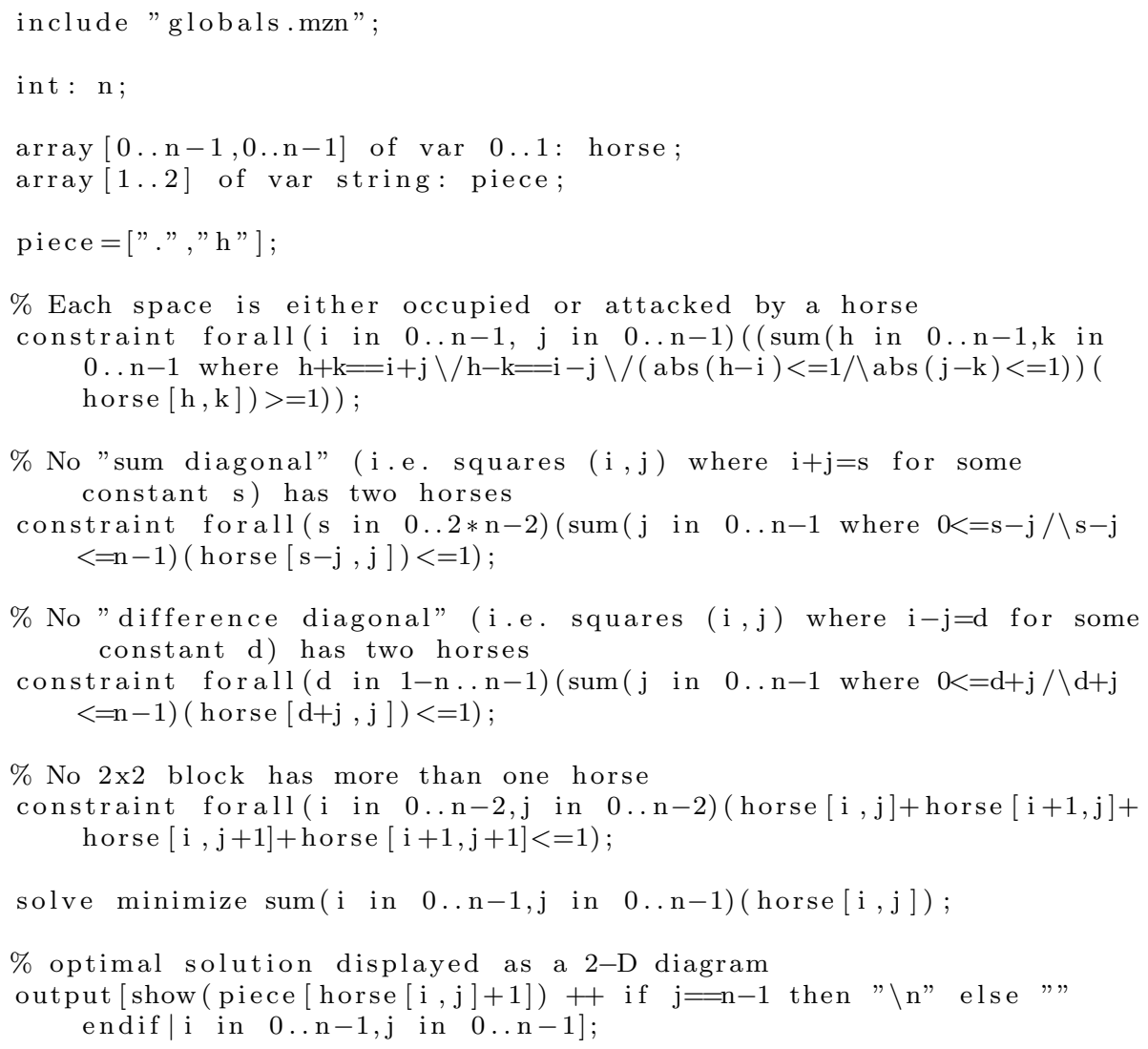

Figure 9: MiniZinc model for determining $i\left(H_{n}\right)$. 
The problem of finding the (independent) domination number of a graph can be expressed as an integer programming problem. (See [4, Section 11.1] and [5. Chapter 1].) We set up models in MiniZinc [7, 9, for the domination and independent domination numbers of $H_{n}$ and applied the G12 MIP solver to those models. The model for $i\left(H_{n}\right)$ is shown in Figure 9] To get a model for $\gamma\left(H_{n}\right)$, take Figure 9 and remove lines $10-11$. The results indicate that for $4 \leqslant n \leqslant 18$, $\gamma\left(H_{n}\right)=i\left(H_{n}\right)=n-1$, except that $\gamma\left(H_{6}\right)=4$, not 5 . (To see $\gamma\left(H_{6}\right) \leqslant 4$, place dragon horses on squares $(1,1),(1,4),(4,1)$ and $(4,4)$.) However, we lack a general proof that $\gamma\left(H_{n}\right)=i\left(H_{n}\right)=n-1$.

\section{Open problems}

The results in this paper provoke many questions, including the following.

1. It is known that there are $2^{n}$ arrangements of $2 n-2$ independent bishops on an $n \times n$ board where $n \geqslant 2$ [10, Theorem 10.2]. How many arrangements are there of $2 n-3$ independent dragon horses on an $n \times n$ board (where $n \geqslant 5)$ ?

2. Is it true that $\gamma\left(H_{n}\right)=i\left(H_{n}\right)=n-1$ for $n>6$ ?

3. For each $0<k \leqslant n$, we define a $k$-step rook on a $n \times n$ board to be a piece that can move up to $k$ squares in a vertical or horizontal direction and a $k$-step bishop as a piece that can move up to $k$ squares in a diagonal direction. Further, we define a $k$-step dragon king to be the combination of the rook and a $k$-step bishop and a $k$-step dragon horse to be the combination of the bishop and a $k$-step rook. What happens to the domination parameters of the $k$-step dragon kings graph $D_{k, n}$ and $k$-step dragon horses graph $H_{k, n}$ as $k$ increases? How quickly do those parameters reach the corresponding parameters of the queens graph?

We note that since $\beta\left(D_{n}\right)=\beta\left(Q_{n}\right)$ and $E\left(D_{n}\right) \subseteq E\left(D_{k, n}\right) \subseteq E\left(Q_{n}\right)$, we have $\beta\left(D_{k, n}\right)=\beta\left(Q_{n}\right)$ for all $k$. On the other hand, for $n \geqslant 5$, $n=\beta\left(Q_{n}\right)<\beta\left(H_{n}\right)=2 n-3<\beta\left(B_{n}\right)=2 n-2$, so we cannot yet determine $\beta\left(H_{k, n}\right)$ for all $k$ and $n$.

4. What can be said about the domination parameters of other shogi pieces? The shogi king, bishop, and rook move like their chess counterparts (ignoring rules about castling and the king avoiding check), and their graphs have been studied [5, Chapter 6]. The other shogi pieces have nonsymmetrical moves. Suppose the initial position of a player's king is in row 0 and that the opponent's king starts in row $n-1$. Then for the pieces on the player's side:

- The lance moves any number of squares forward; i.e., from $(i, j)$ to $(i, j+k)$ for $k \geqslant 1$.

- The shogi pawn moves one square forward; i.e., from $(i, j)$ to $(i, j+1)$.

- The shogi knight leaps to positions that are two squares vertically forward plus one square to the left or right; i.e., from $(i, j)$ to $(i-1, j+2)$ or $(i+1, j+2)$. 
- The silver general moves one square diagonally or one square vertically forward; i.e., from $(i, j)$ to $(i \pm 1, j \pm 1)$ or $(i, j+1)$

- The gold general moves one square vertically, one square horizontally, or one square diagonally forward; i.e., from $(i, j)$ to $(i, j \pm 1)$ or $(i \pm 1, j)$ or $(i \pm 1, j+1)$.

So, if we examine the appropriate directed graphs, what are the domination parameters for these pieces?

5. Since chess queens do not move through other pieces, placing pawns on a board may increase the maximum number of independent queens we can put on that board. In 3 it is noted that the maximum number of mutually nonattacking queens that can be placed on an $n \times n$ board with pawns is $\frac{n^{2}}{4}$ if $n$ is even and $\frac{(n+1)^{2}}{4}$ if $n$ is odd. The argument divides the board into $2 \times 2$ blocks and notes that each block can take at most one queen, regardless of how many pawns are on the board. That argument also works for dragon kings and dragon horses. So, as 3 asks for queens, how many pawns are needed to allow the maximum number of dragon kings or dragon horses on the board?

Acknowledgment: The author wishes to thank Yoshiyuki Kotani of the Tokyo University of A \& T for suggesting the study of the dragon king and dragon horse.

\section{References}

[1] Bell, J., Stevens, B. "A survey of known results and research areas for n-queens", Discrete Math, 309, 1-31, 2009.

[2] Bodlaender, H., Duniho, F. "Shogi: Japanese chess", 2017.

http://www. chessvariants. com/shogi.htm1

[3] Chatham, D. "The maximum queens problem with pawns", Recreational Mathematics Magazine, 3(6), 95-102, 2016.

[4] Haynes, T.W., Hedetniemi, S.T., Slater, P.J. Fundamentals of Domination in Graphs, Marcel Dekker, New York, 1998.

[5] Haynes, T.W., Hedetniemi, S.T., Slater, P.J. (Eds.). Domination in Graphs: Advanced Topics, Marcel Dekker, New York, 1998.

[6] Kosters, W. A. n-Queens bibliography, 2017.

http://www.liacs.n1/home/kosters/nqueens/

[7] Nethercote, N., Stuckey, P.J., Becket, R., Brand, S., Duck, G.J., Tack, G. "MiniZinc: Towards a standard CP modelling language", in: C. Bessiere (editor), Proceedings of the 13th International Conference on Principles and Practice of Constraint Programming, volume 4741 of LNCS Springer, 529-543, 2007. 
[8] Sloane, N.J.A. Sequence A002464 in The On-Line Encyclopedia of Integer Sequences, 2017.

https://oeis.org

[9] Stuckey, P.J., Feydy, T., Schutt, A., Tack, G., Fischer, J. "The MiniZinc challenge 2008-2013", AI Magazine, 35(2), 55-60, 2014.

[10] Watkins, J. J. Across the Board: The Mathematics of Chessboard Problems, Princeton University Press, 2004. 